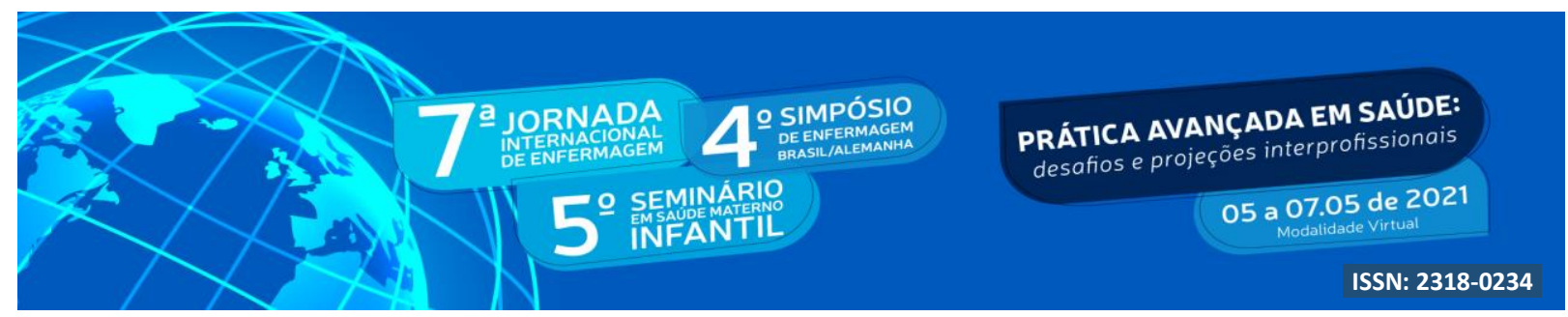

DOI: http://doi.org/10.48195/jie2021-092

\title{
CARACTERIZAÇÃO DO RECÉM-NASCIDO PRÉ-TERMO: DA ALIMENTAÇÃO POR SONDA ATÉ À AUTONOMIA ALIMENTAR ${ }^{1}$
}

\author{
Catarina Sousa² ; Florbela Neto $^{3}$; Ana Paula França4; Margarida Reis Santos ${ }^{5}$
}

\begin{abstract}
RESUMO
Objetivo: Caracterizar os recém-nascidos pré-termo ao nascimento, início da alimentação oral e na autonomia alimentar.

Método: estudo quantitativo e descritivo, realizado numa unidade de cuidados intensivos neonatais de um Hospital Central do Norte de Portugal, aprovado pela Comissão de Ética da instituição.

Colheita de dados realizada entre outubro 2019 e janeiro 2020, por consulta dos registos de enfermagem produzidos entre janeiro 2017 e março 2019. Dados tratados com recurso ao IBM SPSS.

Amostra composta pelos registos de 68 recém-nascidos pré-termo com idade gestacional entre 24 semanas e 4 dias e 35 semanas. No início da alimentação oral a idade dos recém-nascidos variou entre 33 e 35 semanas e na autonomia alimentar entre as 34 semanas e três dias e 38 semanas e cinco dias.

A aquisição de autonomia alimentar do recém-nascido pré-termo é complexa, sofrendo progressos e recuos. Vários fatores influenciam esta transição, como a idade e peso ao nascimento.
\end{abstract}

Palavras-chave: Alimentação; Enfermagem; Recém-nascido pré-termo.

\begin{abstract}
Aim: To characterize preterm newborns at birth, beginning of oral feeding and feeding autonomy.

Method: quantitative and descriptive study, performed in a neonatal intensive care unit at a Central Hospital in Northern Portugal, approved by the institution's Ethics Committee.

Data collection carried out between October 2019 and January 2020, by consulting the nursing records produced between January 2017 and March 2019. Data processed using IBM SPSS. Sample consisting of the records of 68 preterm newborns with gestational age between 24 weeks and 4 days and 35 weeks. At the beginning of oral feeding, the age of newborns varied between 33 and 35 weeks and food autonomy between 34 weeks and 3 days and 38 weeks and 5 days.

The acquisition of food autonomy for the preterm newborn is complex, suffering progress and setbacks. Several factors influence this transition, such gestational age and birth weight.
\end{abstract}

Key Words: Food; Nursing; Preterm Newborn.

\footnotetext{
${ }^{1}$ Estudo descritivo

${ }^{2}$ Mestre em Enfermagem de Saúde Infantil e Pediátrica. Escola Superior de Enfermagem do Porto-ESEP.

E-mail: catarina.sousa239@gmail.com

${ }^{3}$ Assistente convidada. ESEP. Doutoranda em Ciências da Enfermagem ICBAS/UP.

${ }^{4}$ Professora Coordenadora. ESEP. E-mail: apfranca@esenf.pt; ${ }^{5}$ Professora Coordenadora. ESEP.
} 


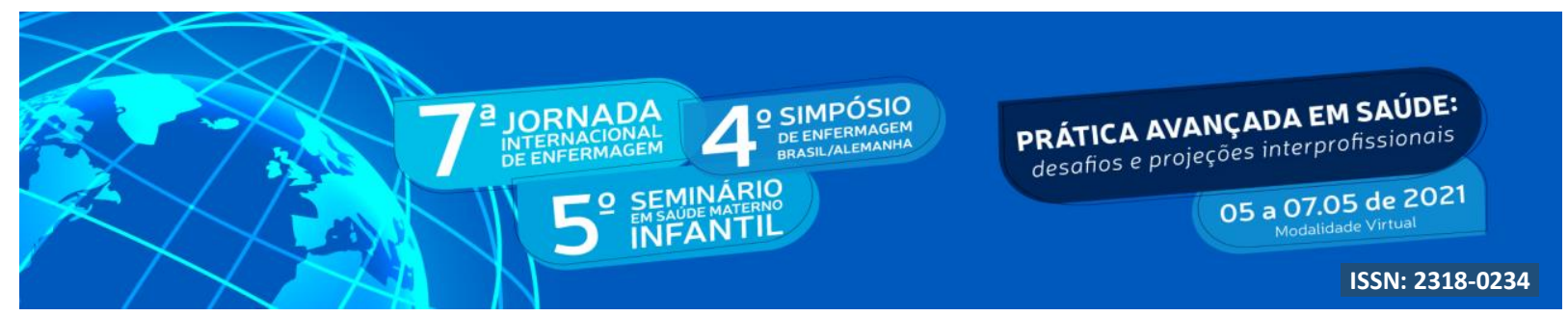

\section{INTRODUÇÃO}

Nas últimas décadas, a sobrevivência dos recém-nascidos pré-termo (RNPT) tem aumentado, principalmente devido à melhoria que se tem vindo a verificar nos cuidados de saúde obstétricos e neonatais. No entanto, os RNPT continuam a ser um grupo vulnerável devido à imaturidade que apresentam no momento do nascimento. Os que nascem antes das 34 semanas de gestação estarem completas apresentam dificuldades a nível da sucção, da deglutição e da respiração. Para que a alimentação dos RNPT seja segura e eficaz é necessária a sincronização destas três funções. Atualmente, a alimentação oral, de acordo com as recomendações das sociedades de neonatologia e a evidência científica, é introduzida entre as 32 e as 34 semanas de gestação, utilizando-se, por vezes, uma abordagem de "tentativa e erro" (LUBBE, 2018; LYU et al. 2020; NETO; FRANÇA; CRUZ, 2016; EMBLETON, 2013).

Os enfermeiros das unidades de cuidados intensivos neonatais (UCIN) desempenham um papel importante na segurança e sucesso da transição para a alimentação oral, devendo ser capazes de avaliar a prontidão para o início da alimentação oral e aplicar intervenções e protocolos, com base em evidências científicas (LAU, 2016; EMBLETON, 2013). Portanto, é crucial que os enfermeiros, tenham conhecimentos atualizados relativamente às competências alimentares do RNPT, de forma a otimizar a segurança e o desenvolvimento de competências destes recém-nascidos, na transição para a alimentação oral (GENNATTASIO et al. 2015; LAU, 2016).

\section{OBJETIVOS}

Caracterizar o RNPT ao nascimento; caracterizar o RNPT à data de início da alimentação oral e caracterizar o RNPT à data da autonomia alimentar.

\section{METODOLOGIA}

Estudo quantitativo, descritivo, retrospetivo, realizado numa unidade de cuidados intensivos neonatais de uma Instituição Hospitalar central do Norte de Portugal, classificada 


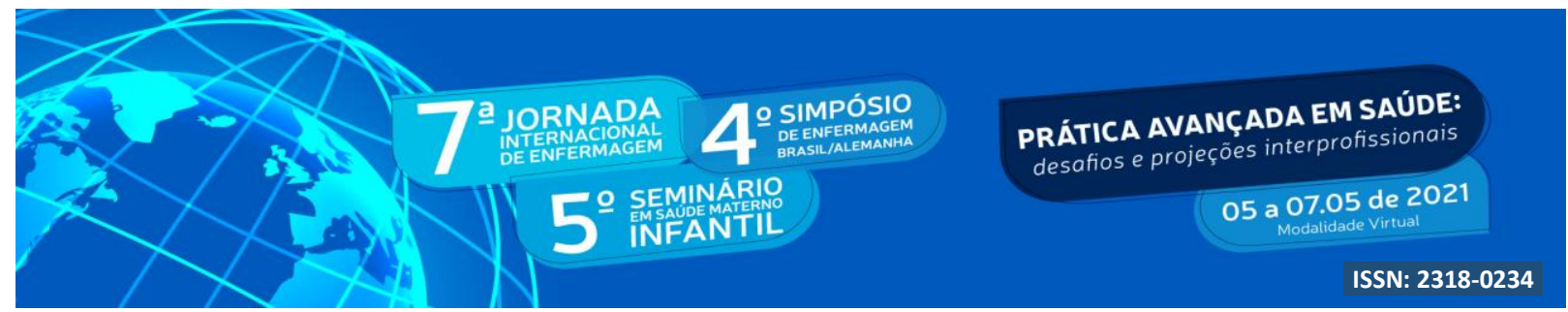

como uma unidade hospitalar de apoio perinatal altamente diferenciado (HAPAD), o que significa que recebe e cuida de recém-nascidos com qualquer tipo de patologia neonatal, desde o nascimento até ao final do período neonatal, nomeadamente cuidados neonatais de subespecialidade específicos, como cuidados pré e pós-operatórios na cirurgia geral neonatal, cuidados pós-operatórios na cirurgia cardíaca ou neurocirurgia, realização de hipotermia e ECMO (ORDEM DOS MÉDICOS, 2013). Desde 2015, esta unidade é um Centro de Formação Newborn Individualized Developmental Care and Assessment Program (NIDCAP).

O estudo foi aprovado pela comissão de ética da instituição (adenda ao protocolo 18819).

A colheita de dados foi realizada através da consulta dos registos de enfermagem produzidos entre janeiro de 2017 e março de 2019, no Sclínico, sistema de apoio aos registos de enfermagem, usado na instituição.

A população-alvo do estudo foi constituída pelos registos de enfermagem referentes aos RNPT internados no serviço de neonatologia da instituição hospitalar selecionada. Os critérios de inclusão na amostra foram os segintes: RNPT que tivessem iniciado a alimentação oral entre as 33 e as 35 semanas de idade gestacional; RNPT que apresentassem estabilidade fisiológica e que fossem alimentados com a totalidade do leite prescrito, por via entérica.

O corpus de análise do estudo constituiu-se por 5451 páginas, referentes aos registos de 146 RNPT, com 35 ou menos semanas de idade gestacional, que estiveram internados (entre janeiro de 2017 e março de 2019) no serviço onde decorreu o estudo. Após análise foram incluídos no estudo os registos de 79 RNPT que cumpriam os critérios de inclusão previamente definidos. Durante o estudo foram excluídos os registos de 11 RNPT, devido a cessação de registos, nomeadamente sobre a autonomia alimentar (8 RNPT); transferência para outro hospital, sem ter atingido a autonomia alimentar (1 RNPT); transferência para o serviço de pediatria do hospital, antes de atingirem a autonomia alimentar (2 RNPT). A amostra final foi, então, composta pelos registos de 68 RNPT que cumpriam os critérios de inclusão.

Desenvolveu-se uma grelha de análise para registar os dados relevantes para o estudo, que foram tratados com recurso ao programa IBM SPSS versão 25.0. Realizou-se uma análise estatística descritiva dos dados com recurso às medidas de tendência central e distribuição de 


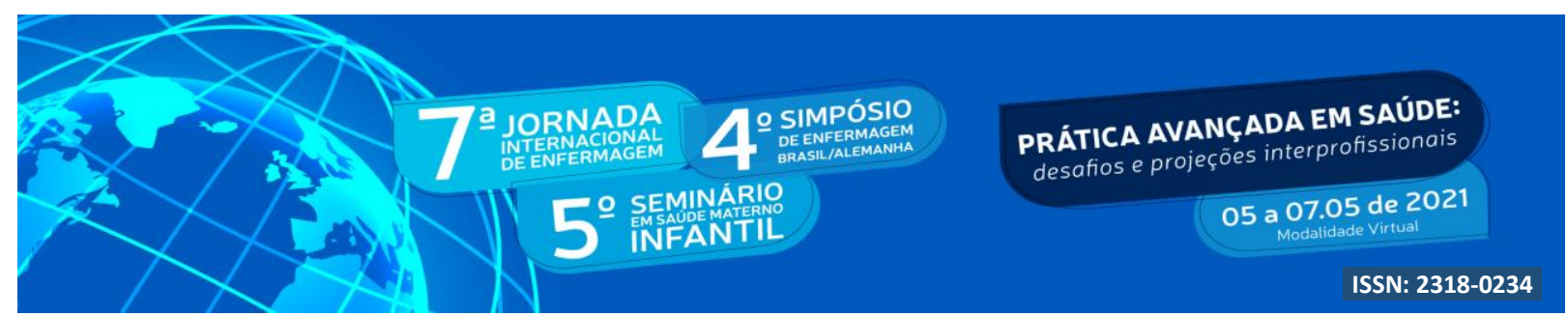

frequências.

\section{RESULTADOS E DISCUSSÃO}

A amostra era homogénea relativamente ao sexo, pois verificou-se que 55,4\% ( $n=37)$ dos RNPT eram do sexo masculino e 45,6\% (n=31) do sexo feminino. Este dado está alinhado com as estatísticas nacionais referentes ao número de nascimentos no ano de 2019, em que nasceram 86.579 crianças, das quais 51,4\% $(\mathrm{n}=44.539)$ eram do sexo masculino (PORDATA, 2020).

O Índice de APGAR ao $1^{\circ}$ minuto, em toda a amostra, apresentou uma moda de 8 , variando entre o mínimo de 3 e o máximo de 9; ao $5^{\circ}$ minuto, apresentou um valor mínimo de 4 e um máximo de 10; ao $10^{\circ}$ minuto, o valor mínimo foi 6 e o máximo 10.

Quanto à idade dos RNPT, mais especificamente às semanas de gestação à nascença, em ambos os sexos, a mediana foi de 33 semanas e um dia e a moda de 34 semanas e cinco dias. Estes dados vão ao encontro da proporção de nados-vivos prematuros, por duração da gravidez, em Portugal, no ano de 2017, em que 8,1\% dos partos totais ocorreram com 36 ou menos semanas de gestação, tendo $7,1 \%$ ocorrido entre as 32 e as 36 semanas de gestação (DIREÇÃO-GERAL DA SAÚDE, 2018). O RNPT com menor idade gestacional à nascença tinha 24 semanas e quatro dias, enquanto o de maior idade gestacional tinha 35 semanas e zero dias. Verificou-se, ainda, que o RNPT com menor idade gestacional à nascença $(24 \mathrm{~s} 4 \mathrm{~d})$ era do sexo masculino (Tabela 1).

Tabela 1: Idade gestacional à nascença, no início da alimentação oral e na autonomia alimentar

\begin{tabular}{|c|c|c|c|c|c|}
\hline \multicolumn{7}{|c|}{ Idade gestacional à nascença } \\
\hline Sexo & $\mathrm{n}$ & Mínimo & Máximo & Mediana & Moda \\
\hline Masculino & 37 & $24 \mathrm{~s} 4 \mathrm{~d}$ & $35 \mathrm{~s}$ & $32 \mathrm{~s} 4 \mathrm{~d}$ & $29 \mathrm{~s} 5 \mathrm{~d}$ \\
\hline Feminino & 31 & $27 \mathrm{~s} 5 \mathrm{~d}$ & $35 \mathrm{~s}$ & $33 \mathrm{~s} 5 \mathrm{~d}$ & $34 \mathrm{~s} 5 \mathrm{~d}$ \\
\hline Ambos os sexos & 68 & $24 \mathrm{~s} 4 \mathrm{~d}$ & $35 \mathrm{~s}$ & $33 \mathrm{~s} 1 \mathrm{~d}$ & $34 \mathrm{~s} 5 \mathrm{~d}$ \\
\hline \multicolumn{7}{|c|}{ Idade no início da alimentação oral } \\
\hline Sexo & $\mathrm{n}$ & Mínimo & Máximo & Mediana & Moda \\
\hline Masculino & 37 & $33 \mathrm{~s}$ & $35 \mathrm{~s}$ & $34 \mathrm{~s} 2 \mathrm{~d}$ & $34 \mathrm{~s} 5 \mathrm{~d}$ \\
\hline
\end{tabular}




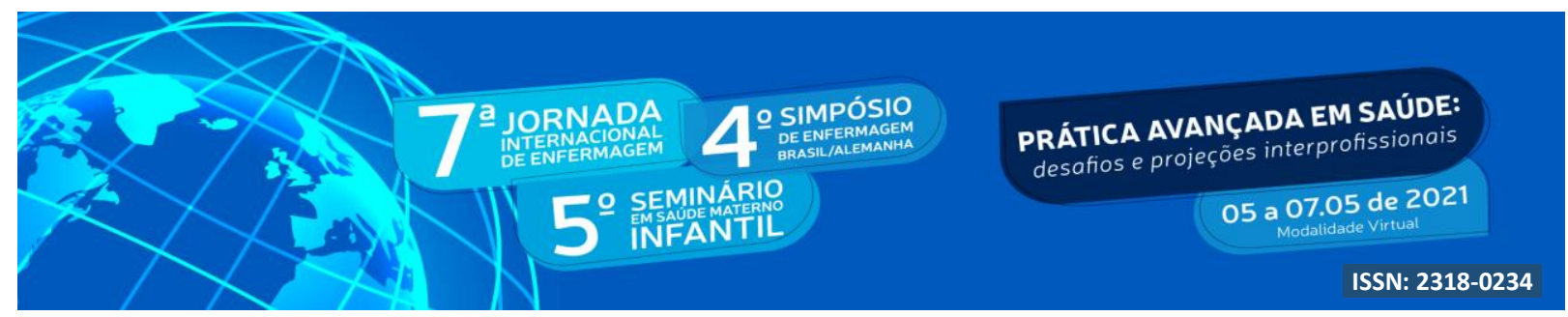

\begin{tabular}{|c|c|c|c|c|c|}
\hline Feminino & 31 & $33 \mathrm{~s}$ & $35 \mathrm{~s}$ & $34 \mathrm{~s} 3 \mathrm{~d}$ & $34 \mathrm{~s} 2 \mathrm{~d}$ \\
\hline Ambos os sexos & 62 & $33 \mathrm{~s}$ & $35 \mathrm{~s}$ & $34 \mathrm{~s} 2 \mathrm{~d}$ & $34 \mathrm{~s} 2 \mathrm{~d}$ \\
\hline \multicolumn{7}{|c|}{ Idade na autonomia alimentar } \\
\hline Sexo & $\mathrm{n}$ & Mínimo & Máximo & Mediana & Moda \\
\hline Masculino & 37 & $34 \mathrm{~s} 3 \mathrm{~d}$ & $37 \mathrm{~s} 4 \mathrm{~d}$ & $35 \mathrm{~s} 5 \mathrm{~d}$ & $35 \mathrm{~s}$ \\
\hline Feminino & 31 & $34 \mathrm{~s} 4 \mathrm{~d}$ & $38 \mathrm{~s} 5 \mathrm{~d}$ & $36 \mathrm{~s} 1 \mathrm{~d}$ & $36 \mathrm{~s} 5 \mathrm{~d}$ \\
\hline Ambos os sexos & 68 & $34 \mathrm{~s} 3 \mathrm{~d}$ & $38 \mathrm{~s} 5 \mathrm{~d}$ & $35 \mathrm{~s} 6 \mathrm{~d}$ & $35 \mathrm{~s}$ \\
\hline
\end{tabular}

Relativamente ao peso à nascença verificou-se uma amplitude entre as $760 \mathrm{~g}$ e as 3280g (M=1683g; DP=484,77; $\mathrm{Me}=1687 \mathrm{~g} ; \mathrm{Mo}=1100 \mathrm{~g})$, quando analisados ambos os sexos. $\mathrm{O}$ peso mais baixo ao nascimento verificou-se no sexo masculino e o mais alto no sexo feminino (Tabela 2).

Tabela 2: Peso à nascença, no início da alimentação oral e na autonomia alimentar

\begin{tabular}{|c|c|c|c|c|c|c|c|}
\hline \multicolumn{7}{|c|}{ Peso à nascença } \\
\hline Sexo & $\mathrm{n}$ & Mínimo & Máximo & Mediana & Moda & Média & DP \\
\hline Masculino & 37 & 760 & 2525 & 1720 & 1100 & 1678 & 453,08 \\
Feminino & 31 & 820 & 3280 & 1670 & 1250 & 1690 & 528,69 \\
Ambos os sexos & 68 & 760 & 3280 & 1687 & 1100 & 1683 & 484,77 \\
\hline \multicolumn{7}{|c|}{ Peso no início da alimentação oral } \\
\hline Sexo & $\mathrm{n}$ & Mínimo & Máximo & Mediana & Moda & Média & DP \\
\hline Masculino & 37 & 1305 & 2525 & 1830 & 2075 & 1847 & 287,07 \\
\hline Feminino & 31 & 1023 & 3280 & 1775 & 1890 & 1794 & 410,85 \\
\hline Ambos os sexos & 68 & 1023 & 3280 & 1817 & 1785 & 1823 & 347,22 \\
\hline Sexo & $\mathrm{n}$ & Mínimo & Máximo & Mediana & Moda & Média & DP \\
\hline Masculino & 37 & 1460 & 2590 & 2150 & 2120 & 2114 & 213,11 \\
\hline Feminino & 31 & 1665 & 3200 & 2050 & 2000 & 2066 & 305,99 \\
\hline Ambos os sexos & 68 & 1460 & 3200 & 2117 & 2000 & 2092 & 258,65 \\
\hline
\end{tabular}




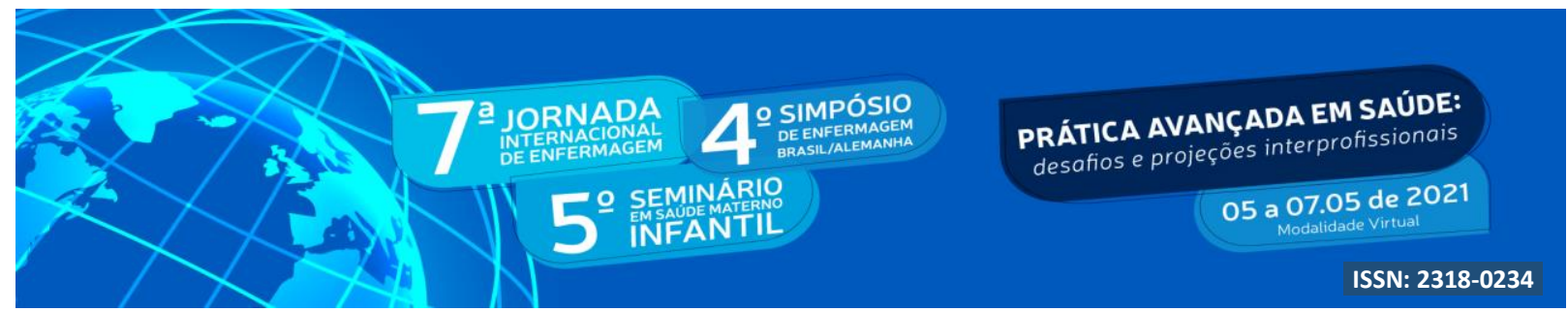

A idade de início da alimentação oral, quando considerados os RN de ambos os sexos, variou entre as 33 semanas e zero dias $(2,9 \% ; n=2)$ e as 35 semanas e zero dias $(14,7 \%$; $\mathrm{n}=10$ ). Os RNPT iniciaram a alimentação oral com um valor de mediana de idade de 34 semanas e dois dias. Este resultado é idêntico ao reportado por outros investigadores (WANG et al. 2018; KAYA; AYTEKIN, 2017). No entanto, é de assinalar que não é consensual a idade para o início da alimentação oral, sendo divergentes as opiniões dos investigadores sobre este assunto.

Relativamente ao peso no início da alimentação, verificou-se uma amplitude entre as $1023 \mathrm{~g}$ e as 3280g, quando considerados os RNPT de ambos os sexos (M=1823g; DP=347,22; $\mathrm{Me}=1817 \mathrm{~g} ; \mathrm{Mo}=1785 \mathrm{~g})$. Os RNPT com menor e maior peso no início da alimentação oral eram do sexo feminino (Tabela 2). Este resultado suporta o reportado por outros investigadores (WANG et al. 2018; KAYA; AYTEKIN, 2017; YAMAMOTO et al. 2017).

A idade com que os RNPT atingiram a autonomia alimentar variou entre as 34 semanas e três dias e as 38 semanas e cinco dias. A moda foi 35 semanas $(8,8 \%$; n=6) e 35 semanas e três dias $(8,8 \%$; $n=6)$. A mediana da idade dos RNPT na autonomia alimentar, em ambos os sexos, foi 35 semanas e seis dias e a moda 35 semanas e zero dias. O RNPT que atingiu a autonomia alimentar numa idade mais precoce era do sexo masculino, enquanto o que a atingiu com maior idade era do sexo feminino (Tabela 1). Wang e outros (2018) e Fontana e outros (2018) obtiveram resultados diferentes, tendo os RNPT que participaram no nosso estudo atingido a autonomia cerca de uma semana mais cedo. Tais diferenças podem ser explicadas pelas características das amostras, uma vez que Fontana e outros (2018) incluíram RNPT com idade gestacional ao nascimento entre as 25 semanas e as 29 semanas e seis dias, enquanto neste estudo a variação foi entre as 24 semanas e quatro dias e as 35 semanas.

Quanto ao peso do RNPT, no momento da autonomia alimentar, constatou-se que o valor médio foi de 2092g (DP=258,65; Me=2117g; Mo=2000g), com uma amplitude entre as $1460 \mathrm{~g}$ (RNPT, do sexo masculino, com 35 semanas de idade) e as $3200 \mathrm{~g}$ (RNPT, do sexo feminino, com 34 semanas e cinco dias de idade) (Tabela 2). Nos estudos encontrados nenhum considerou o peso no momento da autonomia alimentar, apenas o peso no momento da alta (CHORNA et al. 2014; KHODAGHOLI et al. 2018; KAYA; AYTEKIN, 2017; 


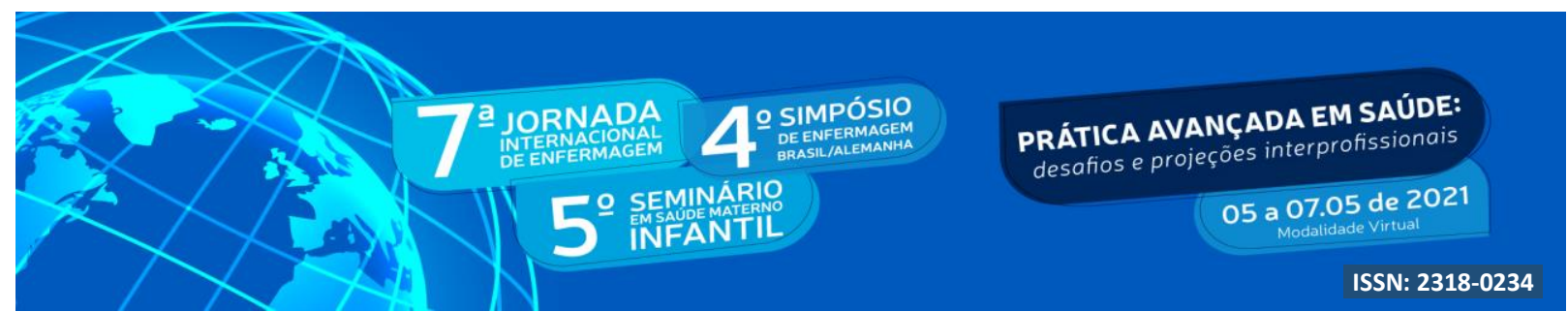

CALIK; ESENAY, 2019; SAY et al. 2018). No entanto, nesta investigação optou-se por verificar o peso no momento da autonomia pois considerou-se ser este um momento chave na transição para a alimentação oral.

Verificou-se, também, que os RNPT demoraram entre um dia $(4,4 \%$; $=3)$ e 38 dias $(1,5 \% ; n=1)$ a alcançar a autonomia alimentar, tendo demorado, em média, 11,84 dias $(\mathrm{DP}=7,47 ; \mathrm{Me}=11,50 \mathrm{~d} ; \mathrm{Mo}=9 \mathrm{~d})$.

Como limitações para este estudo destaca-se alguma escassez dos registos produzidos pelos enfermeiros, o que implicou a perda de alguns RNPT que cumpriam os critérios de inclusão. Sabemos que outras variáveis podem intervir na conquista da autonomia alimentar do RNPT, por esse motivo consideramos que estudos longitudinais e estudos que incluam outras variáveis podem dar informações relevantes para o estudo desta problemática.

\section{CONCLUSÃO}

O processo de aquisição de autonomia alimentar é complexo, muitas vezes com progressos e recuos, sendo vários os fatores que influenciam esta transição, tais como a idade gestacional, o peso ao nascimento, ou a estimulação do RNPT.

As principais conclusões deste estudo revelam que: a idade dos RNPT à nascença, variou entre as 24 semanas e quatro dias e as 35 semanas e zero dias, com um valor de mediana de 33 semanas e um dia; o peso à nascença apresentou o valor médio de $1683 \mathrm{~g}$; os RNPT iniciaram a alimentação oral entre as 33 e as 35 semanas de idade, com um valor de mediana de 34 semanas e dois dias; o peso no início da alimentação oral era em média de $1823 \mathrm{~g}$; os RNPT alcançaram a autonomia alimentar entre as 34 semanas e três dias e as 38 semanas e cinco dias, com um valor de mediana de 35 semanas e seis dias; o peso no momento da autonomia alimentar apresentou o valor médio de $2092 \mathrm{~g}$.

A autonomia alimentar do RNPT traz inúmeros benefícios, quer para o RNPT quer para a sua família, além de ser um dos critérios para a alta hospitalar. Assim, quanto mais rápida e bem-sucedida for a transição da alimentação por sonda para a alimentação oral, mais cedo os RN estarão aptos para irem para casa, diminuindo os riscos associados ao internamento (infeções associadas aos cuidados de saúde, por exemplo). 


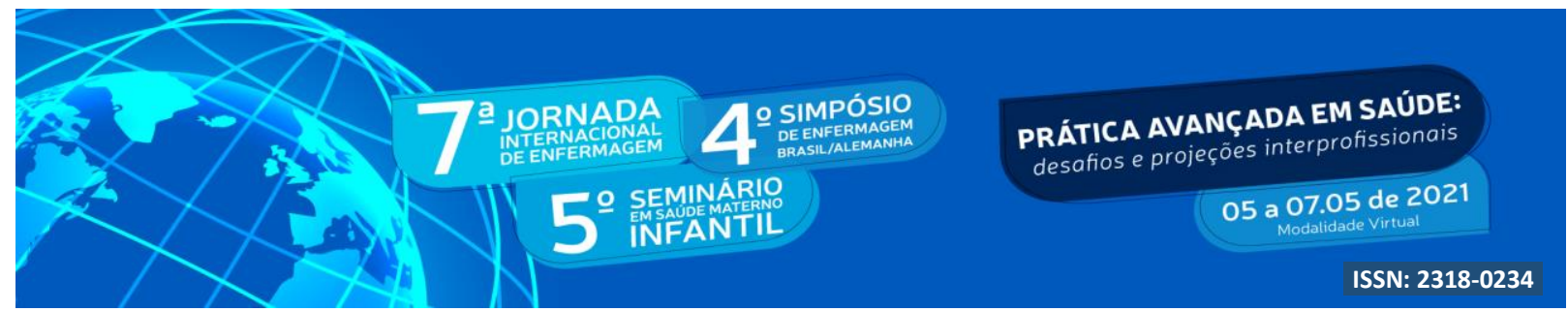

O internamento é, também, uma fonte de stresse para os pais e, consequentemente, para os RNPT. Compreende-se, portanto, que as sequelas da prematuridade não são apenas físicas, mas, também, psicossociais e emocionais, destacando-se o impacto negativo nas famílias: stresse financeiro, familiar, conjugal e individual (GENNATTASIO et al. 2015; KAWAFHA, 2018; TAMEZ, 2013).

Por sua vez, o ambiente da UCIN é agressivo para o RNPT, dado ser um local muito iluminado, barulhento, que expõe os a grande diversidade de estímulos, ou seja, um ambiente totalmente diferente do útero materno que não favorece o seu desenvolvimento. Portanto, a aquisição da autonomia alimentar, de forma segura e eficaz, irá diminuir o tempo de internamento e, consequentemente, a exposição do RNPT ao ambiente da UCIN, contribuindo para que o seu desenvolvimento se continue a processar num ambiente mais adequado, influenciando também a vinculação e a ligação mãe/pai-filho.

O alcance da autonomia alimentar é um dos grandes objetivos para o RNPT e para os profissionais de saúde, nomeadamente para os enfermeiros, ao longo do internamento do RNPT na UCIN. A tomada de decisão para a introdução da alimentação oral deve fazer-se com base na melhor evidência científica, mais do que na experiência dos enfermeiros. Neste sentido, reconhece-se cada vez mais a necessidade de uma prática baseada na evidência e de novos estudos sobre esta temática.

\section{REFERÊNCIAS}

CALIK, Cerean, ESENAY, Figenlsik. The clinical effect of pacifier use on orogastric tubefed preterm infants: A randomized controlled trial. Journal of Pakistan Medical Association, v. 69, n. 6, 2018. Disponível em: 〈https://pubmed.ncbi.nlm.nih.gov/31189280/>. Acesso em: 18 mar. 2021.

CHORNA, Olena et al. A Pacifier-Activated Music Player With Mother's Voice Improves Oral Feeding in Preterm Infants. Pediatrics, v. 133, n. 3, 2014. Disponível em: <https://pubmed.ncbi.nlm.nih.gov/24534413/>. Acesso em: 18 mar. 2021.

DIREÇÃO-GERAL DA SAÚDE. Relatório saúde infantil e juvenil - Portugal 2018. Lisboa: DGS, 2018. Disponível em: <https://www.dgs.pt/documentos-e-publicacoes/relatorio-saudeinfantil-e-juvenil-portugal-2018.aspx>. Acesso em: 18 mar. 2021. 


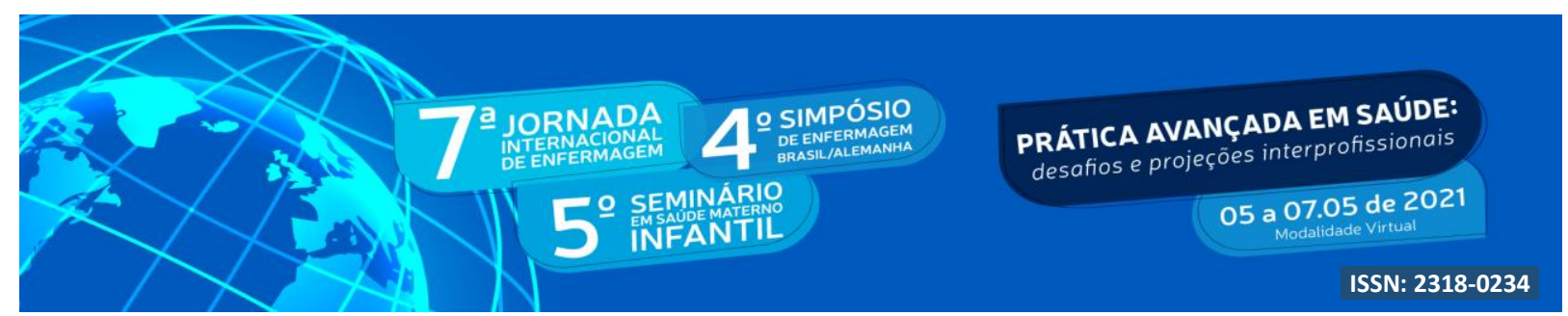

EMBLETON, Nicholas. Optimal nutrition for preterm infants: Putting the ESPGHAN guidelines into practice. Journal of Neonatal Nursing, v. 19, n. 4, 2013. Disponível em: $<$ https://www.researchgate.net/publication/257524065_Optimal_nutrition_for_preterm_infant s_Putting_the_ESPGHAN_guidelines_into_practice >. Acesso em: 18 mar. 2021.

FONTANA, Camilla et al. Effects of early intervention on feeding behavior in preterm infants: A randomized controlled trial. Early Human Development, v. 121, 2018. Disponível em: https://pubmed.ncbi.nlm.nih.gov/29730130/. Acesso em: 18 mar. 2021.

GENNATTASIO, Annmarie et al. Oral Feeding Readiness Assessment in Premature Infants. MCN: The American Journal of Maternal/Child Nursing, v. 40, n. 2, 2015. Disponível em:<https://pubmed.ncbi.nlm.nih.gov/25494013/>. Acesso em: 18 mar. 2021.

KAWAFHA, Mariam. Parental stress in the neonate intensive care unit and its association with parental and infant characteristics. Journal of Neonatal Nursing, v. 24, n. 5, 2018. Disponível em:

<https://www.sciencedirect.com/science/article/abs/pii/S1355184118300383>. Acesso em: 18 mar. 2021.

KAYA, Vildan, AYTEKIN, Aynur. Effects of pacifier use on transition to full breastfeeding and sucking skills in preterm infants: a randomised controlled trial. Journal of Clinical Nursing, v. 26, n. 13-14, 2017. Disponível em: <https://pubmed.ncbi.nlm.nih.gov/27754572/>. Acesso em: 18 mar. 2021.

KHODAGHOLI, Zahra et al. The Effect of Non-Nutritive Sucking and Maternal Milk Odor on the Independent Oral Feeding in Preterm Infants. Iranian Journal of Child Neurology, v. 12, n. 4, 2018. Disponível em: <https://www.ncbi.nlm.nih.gov/pmc/articles/PMC6160630/>. Acesso em: 18 mar. 2021.

LAU, Chantal. Development of infant oral feeding skills: what do we know?1-3. The American Journal of Clinical Nutrition, v. 103, n. 2, 2016. Disponível em: <https://pubmed.ncbi.nlm.nih.gov/26791183/>. Acesso em: 18 mar. 2021.

LUBBE, Welma. Clinicians guide for cue-based transition to oral feeding in preterm infants: An easy-to-use clinical guide. Journal of Evaluation in Clinical Practice, v. 24, n. 1, 2018. Disponível em: <https://onlinelibrary.wiley.com/doi/full/10.1111/jep.12721>. Acesso em: 18 mar. 2021.

LYU, Tianchan et al. Management of Oral Feeding Challenges in Neonatal Intensive Care Units (NICUs): A National Survey in China. Frontiers in Pediatrics, v. 8, 2020. Disponível em: <https://www.frontiersin.org/articles/10.3389/fped.2020.00336/full>. Acesso em: 18 mar. 2021.

NETO, Florbela, FRANÇA, Ana Paula, CRUZ, Sandra. OC24 - An algorithm proposal to oral feeding in premature infants. Nursing Children and Young People, v. 28, n. 4, 2016. 


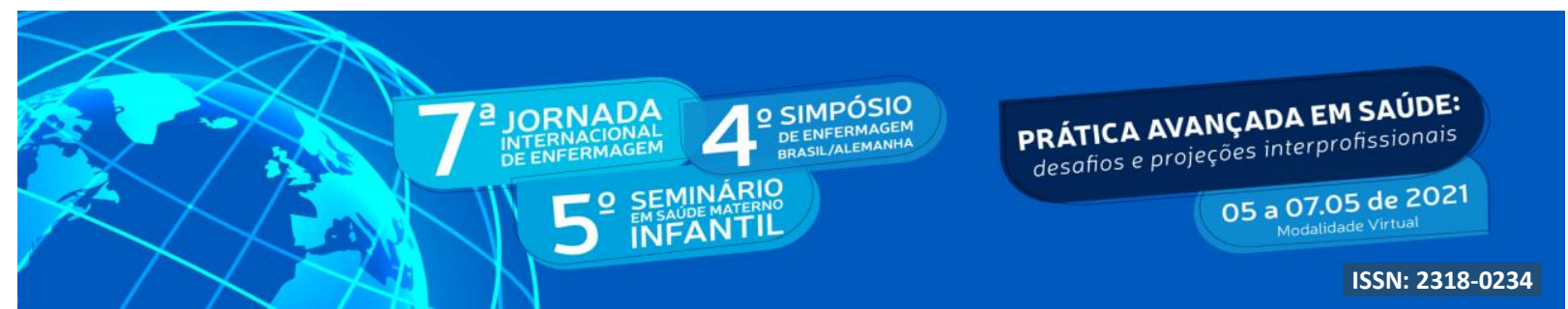

Disponível em: <https://pubmed.ncbi.nlm.nih.gov/27214439/>. Acesso em: 18 mar. 2021.

ORDEM DOS MÉDICOS. Recomendação do Colégio de Neonatologia sobre o número mínimo de Neonatologistas por Unidade de Neonatologia hospitalar. Lisboa: OM, 2013. Disponível em: <https://ordemdosmedicos.pt/wpcontent/uploads/2018/08/Recomenda\%C3\%A7\%C3\%A3o-do-Col\%C3\%A9gio-deNeonatologia-sobre-o-n\%C3\%BAmero-m\%C3\%ADnimo-de-neonato....pdf >. Acesso em: 18 mar. 2021.

PORDATA. Nados-vivos de mães residentes em Portugal: total e por sexo. Lisboa: PORDATA, 2020. Disponível em: https://www.pordata.pt/Municipios/Nados+vivos+de+m\%C3\%A3es+residentes+em+Portugal +total+e+por+sexo-103>. Acesso em: 18 mar. 2021.

SAY, Birgul et al. Effects of Pacifier Use on Transition Time from Gavage to Breastfeeding in Preterm Infants: A Randomized Controlled Trial. Breastfeeding Medicine, v. 13, n. 6, 2018. Disponível em: <https://pubmed.ncbi.nlm.nih.gov/29912580/>. Acesso em: 18 mar. 2021.

TAMEZ, Raquel. Enfermagem na UTI neonatal. 5. ed. Rio de Janeiro: Guanabara Koogan, 2013.

WANG, Yu-wen et al. Effect of a Delayed Start to Oral Feeding on Feeding Performance and Physiological Responses in Preterm Infants: A Randomized Clinical Trial. Journal of Nursing Research, v. 26, n. 5, 2018. Disponível em: <https://europepmc.org/article/med/29401082>. Acesso em: 18 mar. 2021.

YAMAMOTO, Raquel et al. A relação entre saturação de oxigênio, idade gestacional e nível de habilidade de alimentação oral de recém-nascido pré-termo. Codas, v.29, n.1, 2017. Disponível em: <http://www.scielo.br/scielo.php?script=sci_arttext\&pid=S231717822017000100304 >. Acesso em: 18 mar. 2021. 